\section{Effect of Hydroquinone on Mitosis}

IN a previous communication, Parmentier and Dustin ${ }^{1}$ reported a mitotic anomaly in the mouse intestine produced by hydroquinone. The characteristic feature of this was the so-called 'three-group metaphase', comprising a normal metaphase plate with additional chromosome particles or groups at each pole of the spindle. One of the authors afterwards described the same phenomenon in the intestine and bone marrow of the golden hamster ${ }^{2}$. According to the latter, characteristic 'three-group metaphases' may also be produced in the rat, whereas "the guinea pig, the chick and chick fibroblast cultures were not responsive as regards this effect".

We wish to report analogous effects produced by hydroquinone in vitro in chick fibroblasts, and in vivo in the liver, the bone marrow and the cornea of the rat.

Chick fibroblast cultures (third passage) were immersed in a solution of 1 in $10^{-6}$ hydroquinone in Ringer's solution and incubated for one hour, or immersed in a solution of 1 in $10^{-7}$ and incubated for $2 \mathrm{hr}$. The whole cultures were then fixed and stained with Giemsa's stain. Typical hydroquinone mitoses could be observed (Fig. 1).

In rats treated intraperitoneally with the same doses as used by Parmentier and Dustin, we observed, $2 \mathrm{hr}$. after treatment, mitoses in the liver, some showing the characteristic features (Fig. 2) as described by these authors. In the bone marrow the 'three-group mitoses' were found in the white precursors (Fig. 3), whereas the red precursors showed often very marked karyorrhexis. As in the cultures, only a certain number of mitoses showed these changes, others being normal. On the whole, mitoses were not very numerous in the bone marrow after treatment with hydroquinone.

In the cornea of the rat the same effect was seen $2 \mathrm{hr}$. after instillation of one drop of a 5 per cent solution of hydroquinone into the conjunctival sac, or after intraperitoneal injection of hydroquinone in doses mentioned above.

These observations, added to those of Parmentier and Dustin, show that hydroquinone had a general effect on various tissues, manifested by characteristic changes in the morphological aspect of mitosis. The experiments on chick fibroblasts in vitro and on the cornea of the rat in vivo further suggest that the drug acts directly on the cell.

A. Rosin

F. DolJanski

Department of Experimental Pathology and Cancer Research,

Hebrew University-Hadassah Medical School, Jerusalem, Israel.

Aug. 17.

1 Parmentier, R., and Dustin, P., Nature, 161, 527 (1948).

2 Parmentier, R., Nature, 171, 1029 (1953).

\section{Application of Nigrosine to the Study of the Salivary Chromosomes}

IN this work, an alcohol-soluble nigrosine (Edward Gurr) was used, and the stain prepared by Rosen's method $^{1,2}$. The scheme of treatment followed for staining the salivary chromosomes of Drosophila is as follows. (1) Dissection in $\mathbf{4 5}$ per cent acetic acid. (2) Treatment with freshly prepared alcoholic hydrochloric acid during 1-2 min., keeping the glands well covered. (3) The specimen is washed two or three times carefully with distilled water, and a big drop of water is left on the material for $5 \mathrm{~min}$. (4) Drain thoroughly and carefully. (5) Add a drop of stain and put on a cover slip, without squashing. (6) After 10-15 min., squash under blotting paper and immediately put a big drop of glycerine on one edge of the cover slip. (7) The preparation can be studied at once; but after $24 \mathrm{hr}$., the glycerine having penetrated the specimen thoroughly, it is convenient to get rid of excess liquid and make a wax border. Preparations thus obtained last a long time if handled carefully.

With this method the salivary chromosomes are strongly and specifically coloured. Only the outer bands are dyed, except in the heterochromatin regions. But when the preparation is ageing, the interbands become faintly coloured, and the same happens with the cytoplasm.

The greatest interest of this method is in its peculiar mode of placing the chromocentre. In every case studied the chromocentre shows itself as a differentiated and constant structure for each species. Four structural elements can be distinguished : (1) faint

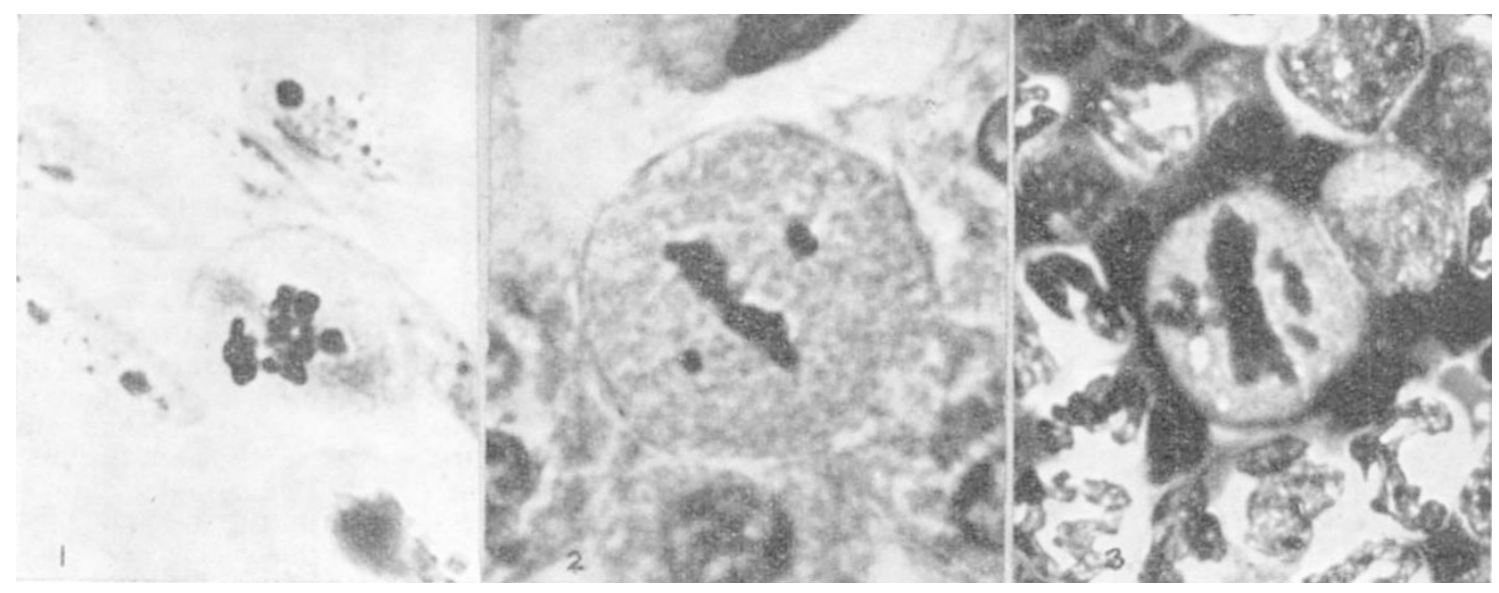

(1) Typical three-group metaphase in chick fibroblast in vitro (Giemsa). $\times 1,600$

(2) The same type of mitosis in liver cell of rat (H.E.). $\quad \times 1,700$

(3) The same in promyelocyte in bone marrow of rat (Giemsa). $\times 1,200$ 